\title{
Comprendiendo las enfermedades raras. Educación y humanismo en la sociedad actual
}

\section{Understanding Rare Diseases. Education and Humanism in Today's Society}

\author{
Anabel Paramá Díaz ${ }^{1}$ \\ Universidad Isabel I (España) \\ ORCID: https://orcid.org/0000-0002-0446-4975
}

Recibido: 06-11-2020

Aceptado: 24-12-2020

\section{Resumen}

El hombre es un ser relacional influenciado por el entorno y por los demás. Una relación que puede ser positiva ya que permite el diálogo, la fraternidad, el cariño y el cuidado, o negativa, porque puede generar sobreprotección, destrucción e incluso violencia. En este sentido, en este trabajo ahondaremos en la comprensión de, precisamente, lo diferente. Para ello nos centraremos en el caso de las enfermedades poco frecuentes y los mecanismos para minimizar los procesos de exclusión que sufre este grupo de personas, la relación con los profesionales de la medicina y aspectos relacionados con el mundo laboral, entre otros. Una serie de aspectos que, debido a la baja prevalencia de estas enfermedades, son poco estudiados, por lo que el avance en el conocimiento de estas patologías se ve muy reducido. Todo esto conlleva graves consecuencias para las personas afectadas, encontrándose desprotegidas en ámbitos como el social y el laboral.

Palabras-clave: Enfermedades raras, discapacidad, teletrabajo, Hermenéutica, riesgos laborales.

\footnotetext{
1 (anaisabel.parama@uil.es) Doctora en Biología. Docente de la Universidad Isabel I. Ha publicado numerosos trabajos en el ámbito de la parasitología y en el contexto de los estudios de carácter social. Algunos de los artículos más destacados han sido publicados en revistas tales como Filosofia. Sociología, Utopía y praxis latinoamericana, Index de enfermería, Parasitology, etc.
} 


\begin{abstract}
The Man is a relational being influenced by the environment and by others. A relationship that can be positive since it allows dialogue, fraternity, affection and care or negative, because it can generate overprotection, destruction and even violence. In this sense, in this work we will delve into the understanding of precisely what is different. For this, we will focus on the case of rare diseases and the mechanisms to minimize the exclusion processes suffered by this group of people, the relationship with medical professionals and aspects related to the world of work, among others. A series of aspects that, due to the low prevalence of these diseases, are little studied so that progress in the knowledge of these diseases is greatly reduced. All this entails serious consequences for the people affected, finding themselves unprotected in areas such as social and labor.
\end{abstract}

Key-words: Rare Diseases, Disability, Telework, Hermeneutic, Occupational Risk Factor.

\title{
Introducción
}

El estudio sobre la importancia de cuidar, de la fraternidad, de la prudencia y de términos que abren la puerta al diálogo y a la comprensión de los demás ha cobrado en los últimos años una especial importancia. El pensamiento iberoamericano se ha convertido, precisamente, en uno de los ámbitos donde ha tenido mayor impacto este tipo de estudios. En este trabajo nos centraremos en el trabajo de algunos de estos autores, tales como Mauricio Beuchot, Ramón Queraltó, Angel Puyol o Josep María Esquirol. No haremos un estudio idiográfico del pensamiento de estos pensadores. Nos interesa más analizar la posibilidad que su planteamiento ofrece a problemas de actualidad como el de las enfermedades raras.

Los conceptos que acabamos de mencionar parten de una concepción del ser humano abierta y relacional (Beuchot, 2015a) donde nuestro entorno y las otras personas nos hacer ser lo que somos. De acuerdo con Coca (2010), la evolución de los diferentes organismos, incluido el ser humano, tiende hacia una creciente relación entre ellos y con el ambiente. Esta idea también ha sido defendida por Queraltó (2003), quien considera que la humanidad encuentra el sentido de su propia existencia, de su vida, en la relación. Relación que, además, abre la puerta a la fraternidad (Puyol, 2017). Una fraternidad política que, junto con la libertad y la igualdad típicas de la Revolución Francesa, configuran un marco democrático claramente humanista. En este sentido Puyol $(2017,43)$ indica lo siguiente: 
El Estado de bienestar recoge el carácter asistencial de la fraternidad, transformado ahora en el ideario de la solidaridad, con el fin de paliar la desigualdad excesiva que padecen los perdedores en el juego del capitalismo, de minimizar la cara agria de un sistema económico despiadado con los que pierden sus oportunidades de éxito social y económico. De este modo, el principio de solidaridad suaviza el temor y la miseria que el capitalismo produce inevitablemente, pero ese fin asistencial está ya muy lejos del objetivo emancipador que la fraternidad revolucionaria también tuvo, según el cual el derecho de existencia no se inspira en la compasión, sino en el principio moral de que la libertad, la igual y la felicidad (...) son derechos naturales e inalienables...

Este carácter democrático y humanista hace que Ángel Puyol acertadamente considere que la sociedad civil será la depositaria de esa fraternidad política nacida en la Revolución Francesa e inherente al ser humano. Esta idea tiene gran potencial, puesto que edifica la agencia social en el marco de una concepción ética de la acción política de los diferentes grupos sociales. Ahora bien, será necesario que se configuren mecanismos educativos efectivos que permitan a la sociedad civil caminar hacia una fraternidad y una relacionalidad más compleja. En ella la diversidad obviamente es un elemento fundamental y estructurante de la sociedad. Sin ella parece poco probable el diálogo y una democracia más desarrollada. Recuérdese que la democracia ha pasado de ser una estructura formal a convertirse en un valor relacionado con la convivencia entre las personas, lo que ha llevado a algunos defensores a apostar por el valor teórico de la democracia. Esta idea está sustentada en la concepción presente en la Revolución Francesa con sus tres pilares fundamentales (Brettschneider, 2006), algo que también defiende Puyol cuando apuesta por la fraternidad política (2017).

Esta relacionalidad educativa y humanista mantiene un poso de respeto a la diferencia, a los matices que cada uno de nosotros poseemos. De ahí que esté estrechamente enlazada con la idea de la mirada atenta que defiende Josep M. Esquirol (2006). Esta mirada atenta es la mirada cuidadora, aquella que centra su interés en la felicidad y bienestar del otro. Nos viene a la cabeza a un padre o una madre que atentamente vigilan a su prole para evitar que se caigan, que se hagan daño, pero sin oprimir ni coartar la libertad. Es por tanto un ejercicio político y ético de respeto basado en el cuidado y, por supuesto, en la fraternidad, de analogía y prudencia. Parece que esta manera de comprender la realidad estaba ya, en cierto modo, en la concepción aurática del Barroco (Soldevilla, 2013). De hecho, decía Esquirol (2006) que este respeto está próximo a la idea de familiaridad e implica el mantenimiento de una distancia respetuosa al otro.

La capacidad de relación que tienen los humanos puede ser positiva o negativa. Positiva porque permite el diálogo, la fraternidad, el cariño y el 
cuidado. Negativa porque puede generar sobreprotección, destrucción e incluso violencia. Por esta razón, una relacionalidad positiva y completa tiene que tener en su interior, como mínimo, los siguientes aspectos fundamentales:

Simhedonia: es un concepto que viene a indicar que cada persona busca la felicidad o el bienestar del otro. Téngase en cuenta que la simhedonia se diferencia de la empatía en que esta segunda centra la relación en el sufrimiento, mientras que la primera en la felicidad y el bienestar ajeno (Jensen 2016). Por otro lado, también es importante tener en cuenta que Royzman y Rozin (2006) estudiando las diferencias entre la simpatía y la simhedonia comprobaron que la simhedonia es más dependiente del apego emocional que la primera. De tal manera que la simpatía es más frecuente en situaciones tensas que la simhedonia.

Fraternidad: es un concepto político el cual mantiene una clara conformación ética y hace referencia a la restauración de las injusticias en el caso que las hubiera (Puyol 2017). Estamos, por lo tanto, ante la otra cara de una moneda donde en un lado está la fraternidad y en el otro la equidad (o si se prefiere la igualdad de oportunidades).

Complejidad: es un término popularizado por Edgar Morin (1997) que indica una aproximación amplia a la realidad. Este enfoque abre la puerta a una concepción no lineal del otro. La relación entre las personas tendrá matices y será diferente en función del contexto, de las características de la propia relación y de la interpretación de los agentes intervinientes. En este sentido, una concepción compleja de la realidad supone la aceptación de la diferencia y, por tanto, de la rareza. Como bien indica Jiménez (2011), la complejidad supone el rechazo a la lógica clásica, según la cual o de da A o se da B. El pensamiento complejo asume la posibilidad de se den ambas (A y B) o ninguna $(\neg A$ y $\neg$ B).

Partiendo de estas ideas básicas, el objetivo del trabajo es ahondar en la comprensión social de las enfermedades poco frecuentes y en los mecanismos que podrían reducir los procesos de exclusión que tienen a su alrededor y especialmente en relación con los aspectos laborales.

\section{Las enfermedades poco frecuentes}

El término de enfermedades poco frecuentes o raras puede hace referencia al conjunto de patologías cuya incidencia en la población es baja. En la Unión Europea se estableció en el año 2009 que una enfermedad será designada como rara cuando su incidencia sea menor de 1 de cada 2.000 personas. Es decir, cuando afecta a menos de 5 de cada 10.000 habitantes. Estos criterios son diferentes en regiones como los Estados Unidos de América. Ahora bien, un buen número de enfermedades raras afectan a muchas menos personas. Estas 
enfermedades se han denominado ultra-raras y afectan a 1 de cada 100.000 habitantes (Palau, 2020). Algunas de ellas, incluso, afectan a 1 de cada millón de habitantes.

El hecho de que el apelativo de raro sea contextual y epidemiológico implica que existan patologías que puedan ser muy frecuentes en determinadas regiones del planeta, mientras que en otras zonas, efectivamente, sean raras. Ahora bien, cuando hablamos de enfermedades raras no suele ser habitual hacer referencias a afecciones que solamente sean raras en una determinada región. Normalmente la designación de rara está vinculada a patologías en su mayoría con un factor causal genético que afectan a poblaciones infantiles (Palau, 2020). Por otro lado, es importante tener siempre presente que las enfermedades poco frecuentes son muy numerosas y diversas. Por ello, epistemológicamente puede generar ciertos problemas utilizar este concepto de manera genérica. Muchas de estas afecciones, además, se manifiestan a modo de síndromes, por lo que una misma enfermedad puede generar diferentes afecciones en los pacientes. Es decir, una persona, por ejemplo, puede ver afectada sus órganos de la visión, mientras que otras ven alteradas su capacidad motórica. Además, también existe la posibilidad de que varias patologías diferentes compartan sintomatologías similares (Ramalle-Gómara et al., 2015), lo cual nuevamente plantea otro problema biomédico, ya que esto dificulta un diagnóstico correcto y, por consiguiente el retraso en el tratamiento más adecuado a la patología. Esta situación de retraso diagnóstico plantea retos políticos de gran calado a los sistemas democráticos actuales. Retos relacionados con la equidad, con la fraternidad, con la distribución de recursos, con el acceso a especialistas conocedores de la enfermedad, etc. Además, también dibuja enormes desafíos a las políticas sociales relacionadas con la dependencia, con la discapacidad, con la diversidad, con la inclusión, etc. Todo ello son temas de gran calado que necesitan mucha investigación. En el presente trabajo no podremos enfrentarnos a todos ellos.

En definitiva, este tipo de enfermedades comparten determinados aspectos biomédicos y sociales. En el primer caso, podemos afirmar que son afecciones crónicas debilitantes a largo plazo, tienen un carácter grave o muy grave ya que pueden ser mortales, necesitan tratamientos costosos, que no tienen que llegar a ser efectivos totalmente, y requieren de cuidados constantes. Todas estas circunstancias ponen de manifiesto problemas psicosociales tanto al paciente como a su familia (Zubizarreta y Ruíz, 2014). Además, estas enfermedades tienen en común que buena parte de ellas pone en una situación social muy compleja a las familias y a los afectados. Complejidad relacionada con la exclusión, la desestructuración social, etc. (Coca, 2019).

De cualquier modo, creemos que alrededor de las enfermedades poco frecuentes, minoritarias o raras, se configura un fenómeno relacional más 
o menos recurrente en todas las afecciones que permiten incrementar el acercamiento y la comprensión de estas enfermedades desde las ciencias humanas y sociales. Este aspecto, así como la importancia del análisis realizado por estas disciplinas científicas, conviene que sea remarcado.

Paramá y Fernández (2014) expusieron que las personas son agentes que pueden impulsar, apoyar y abrir posibilidades a los demás. De hecho, Queraltó (2003) mostró que el ser humano encuentra un sentido a su existencia a través de la relacionalidad. Ahora bien, siempre y cuando la sociedad esté abierta a que los demás desarrollen su propia personalidad y a que evolucionen socialmente con libertad, equidad y fraternidad siempre y cuando no se ejerza sobre nadie la violencia opresiva. En este sentido Esquirol $(2006,61)$ indicó que la violencia consiste en un fenómeno de violación del otro. Este proceso puede ser sobre su cuerpo, sobre su presencia social, sobre su intimidad, sobre aquello que piensa (ideas o creencias), etc. Por tanto, unas personas ejercen violencia sobre otras cuando de algún modo no aceptan la manera en la que otras conforman su realidad personal o social y atentan contra la libertad de estas ejerciendo cualquier posible mecanismo de agresión directo o indirecto sobre ellas.

Desde esta perspectiva, existen grupos sociales sobre los que se generan procesos de violencia por sus condicionantes biológicos, psicológicos o sociales. En este sentido, las enfermedades poco frecuentes se encuentran afectadas por diversos tipos de violencia. Por un lado, nos encontramos con una violencia epistemológica fruto de la carencia de conocimiento existente sobre muchas de las patologías poco frecuentes. En segundo lugar, existe una violencia institucional debido a un proceso de desatención de las necesidades de las personas y familias afectadas. En tercer lugar, también existe cierto grado de violencia en la relación de los demás con las personas afectadas. En este punto existen matizaciones, ya que el rechazo será mayor cuanto más patente sea la manifestación de la enfermedad. Recordemos que, a lo largo de la historia de la humanidad, la enfermedad se ha relacionado a menudo con el contagio. Por lo que existe un sustrato imaginario que identifica enfermedad y posible infección, lo que trae consigo la justificación imaginaria de la violencia del rechazo.

En definitiva, siguiendo el pensamiento de Ramón Queraltó (2003, 271) podemos considerar que la relacionalidad humana tiene una referencia clara en la responsabilidad como valor ante el otro. La violencia es ontológicamente contraria a dicha responsabilidad. No atender las necesidades de otra persona, no preocuparse por otro o evitar el contacto, por ejemplo, son mecanismos violentos en mayor o menor grado, pero violentos, al fin y al cabo. Esto nos lleva a pensar en la situación actual provocada por la pandemia del SARS Cov-2 en la que se fomenta, institucionalmente, cierto ejercicio de violencia al rechazar el contacto con el otro. 
El espacio es, en este sentido, un elemento fundamental en el proceso de diálogo y de ejercicio de una acción de carácter humana y fraterna. En este sentido, Emmanuel Lévinas indica que será en la cercanía del otro donde su mirada cae sobre uno mismo casi de un modo absoluto. En esta cercanía uno, en cierto modo, se desnuda y se convierte en un ser frágil y sin la seguridad de la distancia. Por esta razón Esquirol, tanto en su El respeto o la mirada atenta, como en su Uno mismo y los otros, sigue el pensamiento de Lévinas para establecer una relación humana en la que se establece cierto espacio de libertad y afecto. El espacio justo para que me deje afectar por el otro y para mantener el respeto por esta persona.

Cuando escrutamos con la mirada a una persona afectada por una enfermedad rara que tenga alguna manifestación externa, podemos estar invadiendo su espacio. Es decir, podríamos llegar a ejercer una violencia sobre la persona que se puede sentir despreciada o que puede sentirse agredida. En este sentido Coca (2019) indica que las personas afectadas por estas patologías se sienten marginalizadas $\mathrm{y}$, en el polo opuesto, tratadas con excesivo paternalismo. Por ello, una parte de ellas persiguen la invisibilidad para no ser tratadas de manera diferencial. Por otra parte, siguiendo con el ejemplo de la mirada, si evitamos el contacto con los ojos de la persona enferma, también podemos estar cometiendo cierto grado de violencia, ya que podemos transmitir la sensación de que esa persona es despreciable y, por tanto, no merece ni ser mirada.

En este sentido diversos autores (Coca 2019; von der Lippe 2017) nos muestran que un número importante de personas que sufren algún tipo de enfermedad rara se ha visto afectada por algún tipo de rechazo o exclusión. Este fenómeno lo han percibido, incluso, en el ámbito sanitario. Más concretamente, von der Lippe (2017) indica que, en la mitad de los estudios que analizaron, se afirmaba que las personas afectadas por enfermedades poco frecuentes mostraban una percepción estigmatizante hacia ellos o una concepción negativa como consecuencia de su afección. En algunos casos relacionada con la visibilización de su enfermedad. Este hecho fue constatado en el estudio coordinado por Coca (2019a), donde se comprueba la relación entre las manifestaciones de la patología y el rechazo social. Conviene recordar que, tal y como mostró Sendrail (1983), la sociedad actual se ha convertido en intolerante con aquellos que muestran algún problema de adaptación al sistema establecido. No obstante, Rose (2001) considera que la lógica de la normatividad foucaultiana se está perdiendo y está siendo reemplazada por una lógica de la variación molecular, una modulación de la susceptibilidad e, incluso, de una capitalización de la vida misma. Atendiendo a lo expuesto por Coca (2019a) y por Von der Lippe (2017), entre otros, parece que en el caso de las enfermedades poco prevalentes el peso de la normatividad como elemento 
de segregación se mantiene. Si realmente la variación molecular funcionase, en las relaciones humanas de estas personas, entonces no sería tan habitual la exclusión (especialmente en las patologías en las cuales sus efectos son manifiestos). Por esta razón, y en aras de buscar consensos, podríamos indicar que se están produciendo ambos mecanismos: la normatividad como elemento de exclusión y la variación como elemento explicativo. De cualquier manera, sería conveniente incrementar el proceso de relacionalidad prudente del que hemos hablado antes para minimizar los efectos negativos de las percepciones humanas. Para ello será necesaria una interpretación analógica del otro.

\section{La interpretación analógica del otro}

Mauricio Beuchot es uno de los grandes pensadores actuales sobre el concepto de prudencia y de analogía. Este filósofo mexicano ha propuesto la hermenéutica analógica como un planteamiento humanista que intenta evitar los excesos en los que se encuentra la sociedad actual. Él es consciente que, por un lado, nos hemos vuelto excesivamente rigoristas con las leyes, con los datos y parece que nos cuesta entender que la humanidad no es una máquina. En el polo opuesto nos encontramos con la ausencia de verdad o con la hipertrofia de la subjetividad, enfoques que permiten y admiten cualquier planteamiento, puesto que lo más importante es la vida de cada sujeto y sus percepciones. Ante todo ello, la analogía pretende ser un enfoque donde se mantiene la diferencia (y se defiende), pero sin olvidar el rigor y la verdad.

El profesor Beuchot en su Tratado de hermenéutica analógica concibe su hermenéutica como un planteamiento simbólico, centrado en el límite y cuyo objetivo fundamental es el de la construcción de puentes de comprensión y entendimiento. Estamos, por lo tanto, ante un modelo de interpretación cuyo pilar es el de la fraternidad, lo que implica una clara conexión antropológica y ética. Beuchot estructura un modelo interpretativo con un fuerte carácter mestizo, algo que es fundamental para asentar los pilares de una relacionalidad en la cual la distancia pueda seguir siendo fraterna y, por tanto, ni demasiado alejada, ni demasiado próxima. Mestizaje que implica mezcla, hibridación, relación, dejarse afectar. Mestizaje que es el fenómeno que ha hecho que la humanidad llegue a ser lo que es.

La hermenéutica analógica habla del margen, de la frontera, del límite de nuestra propia humanidad. Uno de los límites más básicos y cercanos es nuestro propio cuerpo y las posibilidades que él nos ofrece (comunicación, percepción, movimiento, etc.). En dicho límite será donde se establezca la relación, en ese mundo del entre del que hablaba Lévinas, en esa zona-región donde el mestizaje comienza ontológicamente. De ahí la importancia que numerosos autores le 
han dado a las manifestaciones emocionales del cuerpo: al beso, a la sonrisa, al llanto, etc. Es decir, será en la dimensión relacional humana donde estén los pilares de las interacciones físicas humanas. Recuérdese que la hibridación, el mestizaje, es ontológico y también es genético, lo que supone la necesidad, por tanto, de contacto físico. Ahora bien, el comienzo de esta futura relación sucede en la zona de intermediación entre las personas. Esta región, estructuralmente hablando, es la sociedad. La zona en la cual acontece la mayor parte de la vida humana y donde también suceden los fenómenos de crisis social y del ejercicio del poder.

Hemos dicho que Mauricio Beuchot apuesta por una interpretación analógica de la humanidad. Ello le conduce a asumir esta relacionalidad sin exclusiones, defendiendo al contrario la equidad en la diversidad. Esta hermenéutica iberoamericana evita los mecanismos de poder basados en visión reductivas de las personas (univocidad), pero también evita caer en relativismos extremos, en los cuales cualquier comportamiento o posicionamiento es admitido (equivocismo).

Pensemos por un momento en la relación médico-paciente. Como resulta evidente, la mayoría de las personas enfermas tienen desconocimiento de su enfermedad y de las prácticas que deben realizar o los medicamentos que deben tomar para mejorar. Estamos, pues, ante una relación en la que el poder está situado a un lado de la balanza (el profesional de la medicina). Dicho poder se encuentra anclado a las capacidades científicas y tecnológicas que estas personas tienen. El problema es que la medicina, como muchas otras ciencias, ha dejado a un lado la razón científica explicativa (la posibilidad de establecer teorías sobre la enfermedad o sobre la patología) y ha optado por la razón tecnológica como elemento principal de su evolución como disciplina científica. Ahora bien:

[...] la racionalidad tecnológica deviene finalmente una voluntad de poder. Rota la razón teorética, perdida su unidad construida por el pensamiento moderno, con una ciencia plagada de soberbios "fragmentos" cognoscitivos pero aislados los unos de los otros, sin ningún horizonte epistemológico claro de convergencia entre los distintos campos del conocimiento, ¿qué es lo que todavía se puede intentar? La respuesta no es difícil: la adaptación más perfecta del mundo al hombre mediante su transformación y su manipulación ya que poseemos los instrumentos necesarios y suficientes para tal fin. La tarea del hombre es entonces voluntad de poder sobre la realidad (Queraltó, 2003: 151 y ss.).

Estas palabras de Queraltó (2003) son especialmente interesantes puesto que subyace a ellas la manipulación de la realidad a lo humano. El problema es que este ejercicio de poder sobre la realidad también puede ejercerse sobre los otros. En tal caso el ser humano termina siendo una tarea más que tendrá que 
ser ajustada a un ideal de realidad. Ideal que, por otro lado, se suele ajustar con la normalidad entendida como eje unívoco de valoración. El profesional de la medicina se sitúa, en la relación con el paciente, por encima de éste (al tener, en principio, mayor conocimiento) y establece una interpretación tendente a la univocidad. Se genera, entonces, una relación de poder en la que el profesional de la medicina establece la norma, la pauta de comportamiento y, además, tiene la capacidad legal de determinar si esta persona esta enferme o no y, de ser así, qué enfermedad tiene. Dicho de otro modo, el profesional de la medicina tiene el poder de transformar la realidad de las personas. En el lado contrario tenemos, como es obvio, al propio enfermo, quien interpreta subjetivamente las palabras de profesional de la medicina. Estamos ante el polo de la equivocidad.

La persona enferma, a través de mecanismos de presión, es capaz de interferir -en cierto modo- en las decisiones del profesional. Tanto es así que autores como Guerrero y Magallanes (2019) indican que en la actualidad se está produciendo un fenómeno de farmacolización de la sociedad. Este contexto de farmacolización social es promovido, por lo menos en parte, por la insistencia y la necesidad que perciben los enfermos de medicarse, por lo que se insta a los profesionales de la medicina a que les receten fármacos (Abraham, 2010). Además, y debido al proceso de interpretación subjetiva de las prescripciones, normas y diagnósticos médicos, las personas enfermas van a comprender la realidad médica a su manera. Lo cual puede traer consigo efectos colaterales. Al fin y al cabo el proceso de diagnóstico está muy relacionado con el diálogo, aunque en los últimos tiempos las pruebas analíticas (denominadas todavía complementarias) han logrado tener una enorme importancia. Ello muestra, precisamente, este juego permanente entre la univocidad y la equivocidad en la relación entre los profesionales de la medicina y los pacientes (o sus familias).

En el caso de las enfermedades raras la situación se está transformando sustancialmente. Coca (2019b) muestra que una gran parte de las personas con enfermedades poco frecuentes están relacionadas con asociaciones de pacientes. Estas asociaciones suelen ser específicas y relacionadas con un tipo de patología más o menos concreta. Además, estas asociaciones suelen realizar congresos, seminarios o jornadas de formación científica e invitan a profesionales expertos en su patología o en temas afines. Por último, y debido a la carencia de información, es habitual que estas personas estén altamente informadas en los aspectos relacionados con su dolencia. Este hecho ha generado una transformación en la relación descrita antes. Ahora estas personas se sienten empoderadas y con capacidad para discutir al profesional de la medicina sobre su situación o la de sus familiares. El profesional, además, puede presentar un mayor o menor desconocimiento de la patología en cuestión. En esta situación la relación deja de ser tan vertical (como era habitual tradicionalmente) y pasa a ser más horizontal o, incluso, se invierte. En este caso 
parece necesario desarrollar mecanismos analógicos de comprensión de esta nueva realidad. Estos mecanismos, como ya hemos dicho, están relacionados con la prudencia y la fraternidad. Ambos polos de la relación (profesional de la medicina y paciente) necesitan hacer un esfuerzo de comprensión del otro. Por esta razón, consideramos de gran importancia la introducción de la dimensión hermenéutica en las ciencias de la salud y del cuidado (hablamos de la biología en sus variantes, de la medicina, de la ética y de la filosofía de la salud, de la sociología de la salud, de la enfermería, etc.), en la que las metáforas, el lenguaje, los conceptos y los símbolos ocupan un lugar central en este tipo de conocimiento (Eriksson 2002). La hermenéutica, entonces, nos abre las puertas a los aspectos profundos de la enfermedad; más, si cabe, la hermenéutica analógica. En ella, lo simbólico nos permite comprender al otro, establecer un diálogo donde la evidencia va más allá del dato científico (Eriksson 2010) y ahondar en el mundo de la vida de las personas del que tanto se habló desde la fenomenología.

La perspectiva hermenéutica presenta una connotación ética ineludible. Al fin y al cabo, interpretar es construir, en cierto modo, el mundo objetivo como una realidad vivible y asumible. De ahí que esta realidad se relacione con aquello que es concebido como bueno (o malo), puesto que lo bueno se intentará favorecer y lo malo no. Lo cual nos conduce a reflexionar sobre lo que deseamos las personas, sobre nuestros fines en la vida y sobre la propia sociedad. En este sentido Ramón Queraltó defiende un planteamiento plural en el que el desarrollo de la persona y su felicidad (Queraltó, 2008a y 2008b), así como la realización humana, tienen una especial importancia. Ello nos acerca, además, a la ética política de Angel Puyol y a la ética relacional de Esquirol, a los que ya hemos citado anteriormente. De hecho, Queraltó (2003) conecta su propuesta ética con los Derechos Humanos con el objetivo de que la ética mantenga un carácter pragmático, al tiempo que sea operativa política y socialmente.

\footnotetext{
Así, ser defensor de los derechos humanos y de la dimensión personal implícita en ellos es importante no sólo porque se esté defendiendo algo bueno, sino porque se está defendiendo algo conveniente, o sea, que reporta beneficio y bien-estar para la vida humana, tanto a nivel individual como colectivo (Queraltó 2003, 281).
}

En este sentido, Queraltó (2010), siguiendo la filosofía pragmática, concibe la ética como la búsqueda de aquellas consecuencias positivas para el ser humano. Por esta razón, evita los planteamientos éticos más inmovilistas y apuesta por una ética pluralista en la que sea posible relacionar planteamientos que, en ocasiones, puedan parecer contrapuestos. Entonces, diríamos nosotros, cabría conjugar visiones tan polarizadas, en principio, como la utilitarista y 
la personalista. No es el momento de adentrarnos en estas cuestiones, así que volvamos de nuevo al pragmatismo de Queraltó. En este sentido Peirce (19311958) considera al pragmatismo como un método por el cual el significado de una concepción está relacionado con las consecuencias prácticas de dicho término o aserto. Necesitamos de la experiencia para saber qué es lo que reporta beneficio a la humanidad. De ahí que Queraltó apueste, como hemos visto, por la defensa de los Derechos Humanos como elementos éticos con un claro beneficio pragmático. De hecho, la ética pluralista que este pensador defiende se basa en un criterio típicamente pragmático: si un artefacto ético permite encauzar la vida de las personas y alcanzar su bienestar existencial, "entonces no hay nada que objetar desde un punto de vista pragmático" (Queraltó 2010, 247). Por lo tanto, el valor estará también delimitado por aspectos filosóficos de carácter pragmático:

\begin{abstract}
Pragmáticamente considerado, algo tiene valor cuando sirve para resolver problemas o conflictos. Ante tal o cual situación de ese estilo, se busca superarla mediante la aplicación de algo que la aminore o incluso que la cancele en cuanto conflictiva. Y ese "algo" es lo que tiene valor. De ahí que la noción pragmática del valor pueda enunciarse entendiendo a éste como pauta de resolución de problemas. El valor, así, se "justificaría" en la medida en que se introduce en el seno del problema o conflicto y lo hace disminuir o desaparecer. Por eso, el valor "vale" en tanto resuelve problemas (Queraltó 2008a, 174).
\end{abstract}

En este punto es notorio ver que este axiólogo se aproxima a Larry Laudan (1977), quien consideraba que el progreso de la ciencia está motivado por una búsqueda de soluciones a los problemas de la sociedad, de las instituciones, etc. Por esta razón, las acciones que conduzcan a solucionar los problemas de las personas con enfermedades raras tienen valor, ya que solventan los problemas de estas personas. Ahora bien, podríamos pensar: si todas las personas tienen problemas éticos, las acciones conducentes a resolver tales problemas serían igualmente valorables, por lo tanto sería más razonable solucionar los problemas que afecten a un mayor número de personas. Desde esta perspectiva cabría la posibilidad de dejar al margen de la satisfacción de las necesidades fundamentales a las personas con enfermedades poco prevalentes.

Esto es precisamente lo que está sucediendo en la actualidad. Las enfermedades raras, por su baja prevalencia, no están siendo muy estudiadas. Ello reduce sustancialmente el avance del conocimiento sobre estas patologías y sobre sus posibles consecuencias en las personas afectadas. Ante esta situación consideramos vital establecer una hermenéutica de carácter político. 


\section{Hermenéutica y política}

La Hermenéutica analógica de Mauricio Beuchot (2016), como él indica, es una política de la interpretación puesto que su objetivo fundamental es la transformación social. Una interpretación políticamente centrada en la ruptura de los totalitarismos indiferenciados y en la ruptura de la fragmentación. Estamos, por lo tanto, ante un pensamiento político basado en la frónesis o en la analogía. Por esta razón, la interpretación política y analógica del mundo necesita de un correlato educativo que permita el rigor y la apertura sin hipertrofiar ninguno de los dos aspectos.

En este sentido, la interpretación política y analógica de la realidad se asienta en un planteamiento crítico al concepto de lo normal como constructo normativo. Benditt (2007) nos muestra que el código normalidad/anormalidad está referido a la función/disfunción molecular, celular, tisular u orgánica. El problema es que, como indica Jutel (2006), se ha configurado como una especie de idea platónica que ha terminado estructurando a la práctica profesional médica. Con anterioridad Rosenberg (2002) indicó que el proceso de diagnóstico médico supone un debate social de gran relevancia. De hecho, esta ideación configura una dificultad para encajar a la humanidad, con sus particularidades, disfuncionalidades $\mathrm{y}$, si se nos permite, rarezas, dentro de ese patrón ideal de lo normal. Además de esto Jutel (2006), en un estudio sobre la obesidad, expone que la transformación de la significación de la obesidad es debida a dos creencias inmersas en el sistema biomédico. Por un lado, la creencia de la neutralidad de la cuantificación y de la objetividad de la medición. La segunda, en cambio, tiene que ver con la importancia de la normatividad en la salud. Estos dos aspectos ayudan a comprender alguno de los elementos que condicionan lo que podríamos denominar como la mitologización de la norma en el ámbito profesional de la medicina en dos sentidos: como normalidad y como normatividad. Ello presenta dificultades para las personas con enfermedades raras a la hora de que los profesionales de la medicina comprendan la situación por lo que estas personas están pasando. En este sentido, consideramos que Josep María Esquirol (2006) puede ayudar al considerar que el respeto es fundamental en el desarrollo ético y, pensamos nosotros, a nivel político. El ethos respetuoso, necesita de la fraternidad política y también de una mirada analógica y prudente. En esta perspectiva ética la rareza (la no normalidad) se convierte en un elemento ético. El ser humano, por tanto, será comprendido en su diversidad, con sus matices y particularidades. De ahí que todo problema biomédico será investigado, comprendido y analizado en equidad. Decimos que, en equidad, ya que la fraternidad política impide que un grupo de personas, por pequeño que sea, quede olvidado o desplazado de la agenda política. Por lo tanto, las instituciones necesitan configurar mecanismos de atención a los 
diferentes colectivos para igualar las posibilidades. Las cuales, actualmente, están limitadas en las personas afectadas por enfermedades poco frecuentes y por sus familias.

En este sentido, Paramá y Fernández (2014) indican que los profesionales sanitarios, como resulta evidente, tienen un respeto propio de su actividad laboral. Ahora bien, en este trabajo hablamos de un ethos que va más allá del respeto propio de las normas educativas colectivas (lo que habitualmente denominamos buena educación). Lo que planteamos es una comprensión más profunda, por parte de los profesionales de la medicina, de las transformaciones que traen consigo las enfermedades poco frecuentes, y que transcienden con mucho los elementos puramente biomédicos. Defendemos, por lo tanto, una equidad en el diálogo en el sistema sanitario que permita generar una transformación política que posibilite la agencia de las personas afectadas en el sistema biomédico.

\section{Las ER en el mundo laboral}

Partiendo de esta perspectiva ética, mencionada anteriormente, nos encontramos con un segundo escalón: la analogía o la fraternidad en el ámbito laboral. Sabemos que las personas con enfermedades poco frecuentes tardan mucho tiempo en recibir el diagnóstico de su enfermedad. Datos obtenidos a partir del Estudio EnSerio (Huete et al, 2009) muestran que, en España, desde que se produce la manifestación de la sintomatología hasta el diagnóstico de la enfermedad, pueden transcurrir entre 5 y 7 años, pudiendo llegar en el 21,01\% de los casos incluso a 10 o más años.

Ello implica que los afectados se pueden encontrar en una situación de espacio vacío laboral y legal. El empleador no tiene una norma que le obligue a realizar una determinada acción o práctica laboral para mejorar la situación de una persona que, biomédica y administrativamente, no tiene un diagnóstico. Todo esto dificulta la inserción laboral de aquellos que padecen este tipo de enfermedades.

Con la situación generada por la pandemia del SARS CoV-2, el teletrabajo ha adquirido una importancia inusitada hasta ahora. Ahora bien, no siempre está bien claro qué significa este concepto. El teletrabajo puede ser definido como cualquier actividad de carácter laboral que no se realice de manera presencial en las instalaciones de la empresa a la que pertenezca el empleado. Para ello, el trabajador tendrá que emplear las nuevas tecnologías o las tecnologías de la información y de la comunicación para su desarrollo (Vicente et al, 2018). En este sentido, López y Chicano (2017) indican que las personas con enfermedades raras, con enfermedades mentales y las mujeres con discapacidad pueden incorporarse al mercado de trabajo con mayor facilidad gracias al 
propio teletrabajo. Ahora bien, para ello es fundamental que esta modalidad de trabajo esté bien diseñada y que se establezcan las medidas oportunas para que la accesibilidad universal en el trabajo funcione adecuadamente. Esta opción laboral eliminaría, por ejemplo, la dificultad que tienen algunas de estas personas para trasladarse al puesto de trabajo y podrían disponer de una mayor flexibilidad en cuanto a horarios (McNaughton et al, 2014).

Esto supondría, por tanto, una mayor oportunidad laboral para las personas con algún tipo de discapacidad permitiendo integrarlas en el mundo laboral (Vicente et al, 2018). Algo a tener en cuenta, pues el 65\% de las personas que sufren algún tipo de enfermedad rara está desempleada (FEDER, 2018).

No obstante, es también relevante tener presente, y más en el contexto social actual, que el teletrabajo puede generar una serie de riesgos potenciales que, muchas veces, no son tenidos en cuenta, tales como el aislamiento social (Mercader, 2016). De ahí que, si las personas que padecen algún tipo de enfermedad rara ya sufren de aislamiento social debido a la exclusión social, a las discapacidades que pueda generar la propia patología o sus propias limitaciones vitales (en sentido amplio), con esta modalidad de trabajo este sentimiento podría llegar a incrementarse todavía más. Lo que plantea la necesidad de crear políticas que vayan encaminadas hacia el fomento de la creación de entornos de trabajo inclusivos para estas personas, de manera que les permitan reducir al máximo posible este aislamiento social a mayores (Baker, Moon y Ward, 2006).

Este planteamiento se encuentra con un problema, lo mencionábamos previamente, y es que el diagnóstico definitivo de la enfermedad se dilata durante varios años, por lo que, si no hay diagnóstico, a nivel legal no hay enfermedad y, por tanto, no tiene por qué darse el caso de alguna transformación del puesto de trabajo o el proceso de adaptación a la enfermedad. Lo que puede llegar a provocar un empeoramiento de la enfermedad.

Las personas que sufren enfermedades de baja prevalencia presentan otro gran escollo laboral. Y es que, tal y como ha mostrado García-Gómez (2014), para determinar la relación entre una determinada patología (sea la que fuere) y el trabajo, resulta necesario tener en cuenta la intensidad de la asociación entre el riesgo potencial y la propia enfermedad. Por otro lado, también es necesario conocer la especificidad inherente al fenómeno, el factor temporal, el gradiente, etc. Toda esta información puede ser desconocida o tener un acceso enormemente complicado. Recuérdese que, al hablar de enfermedades raras, hablamos también de enfermedades sin diagnóstico o de aquellas de las que no se conoce demasiado. Todo esto hace que las personas que sufren enfermedades poco frecuentes y las que sufren enfermedades crónicas sin diagnóstico presenten una capacidad menor para poder llevar a cabo la realización de un trabajo en un tiempo concreto y determinado. 
Hay que tener en cuenta, también, que hay enfermedades que presentan síntomas clínicos inespecíficos y varían en función de la edad de la persona afectada. Este es el caso de, por ejemplo, la neumonía crónica lipoidea. Todo ello conlleva problemas de carácter legal y administrativa, puesto que si no existe determinación de una patología concreta, las personas afectadas carecen de mecanismos mediante los cuales puedan reclamar sus derechos. Por tanto, el ethos planteado también abre la puerta a una mayor comprensión de las dificultades laborales de las personas afectadas por enfermedades raras. En tal caso, el profesional de la medicina -y desde nuestra perspectiva- podría operar como puente para aumentar el sostén social de las personas afectadas en el ámbito laboral.

\section{Conclusiones}

De cualquier modo, la argumentación que aquí se está proponiendo necesita de un correlato educativo. La educación tiene que ser parte de esta transformación ética de la que estamos hablando. Una transformación de carácter pragmático y analógico. Este potencial acción educativa todavía está naciendo y precisa de un posterior trabajo investigador que implemente herramientas y estrategias de aprendizaje formal e informal.

A nivel laboral uno de los principales problemas es la falta de diagnóstico. Constituye la base para poder actuar a nivel legal en el ámbito laboral, de forma que las personas que padecen de enfermedades raras o poco frecuentes puedan ser consideradas a la hora de la adaptación de los puestos de trabajo o cambios en los mismos que les permitan realizar las tareas de una forma menos perjudicable para ellas. 


\section{Referencias bibliográficas:}

Abraham, J. (2010). Pharmaceuticalization of Society in Context: Theoretical, Empirical and Health Dimensions. Sociology, 4(4), 603-622. doi: $10.1177 / 0038038510369368$.

Benditt, T.M. (2007). "Normality, disease and enhancement". In: Kincaid, H. and McKitrick, J. (eds.) Establishing Medical Reality. Dordrecht, Springer: 13-21.

Beuchot, M. (2015a). Antropología filosófica. Hacia un personalismo analógico-icónico. México, Universidad Anáhuac México Sur.

Beuchot, M. (2015b). Tratado de hermenéutica analógica. Hacia un nuevo modelo de interpretación. UNAM, México.

Beuchot, M. (2016). Hechos e interpretaciones. Hacia una hermenéutica analógica. México, FCE.

Brettschneider, C. (2006). The value theory of democracy. Politics, Philosophy \& Economics, 5(3), 259-278. https://doi.org/10.1177/1470594X06068300

Coca, J. R. (2010): La comprensión de la tecnociencia, Hergué, Huelva.

Coca, J. R. (2012): “Utilidad de la hermenéutica analógica para la gestión del conocimiento tecnocientífico". En: Conde Gaxiola, N. (Comp.) Hermenéutica, analogía y mundo actual, Editorial Torres Asociados, México, pp. 153-164.

Coca, J.R. (2019) (eds.) Enfermedades raras. Contribuciones a la investigación social y biomédica. Soria, Ceasga-Publishing.

Coca, J. R. y Valero Matas, J. A. (2012). La «exclusión» cientifica del otro. Ediciones de la Torre, Madrid.

Eriksson, K. (2002). "Caring Science in a New Key". Nursing Science Quarterly 15(1): 61-65.

Eriksson, K. (2010). "Evidence: To See or Not to See". Theoretical Concerns 23(4): 275-279. https://doi.org/10.1177/0894318410380271

Esquirol, J. M. (2006). El respeto o la mirada atenta, Gedisa, Barcelona.

Esquirol, J. M. (2005). Uno mismo y los otros, Herder, Barcelona.

García Gómez, M. (2014). "La sospecha de enfermedad profesional: programas de vigilancia epidemiológica laboral". Medicina y Seguridad del Trabajo, 60(Supl. 1): 157-163.

Guerrero Mondaca, J., y Magallanes Rodríguez, A. (2019). Mexican Men Meet Cyborg Masculinity: Gendered Subjectivities in the Technology of Erection Era. In: B. Braid y H. Muzaffar (eds.) Bodies in Flux. The Netherlands: Rodopi. https://doi.org/10.1163/9789004408760 007 
Huete García, A., Díaz Velázquez, E., Díaz García, E. Sola Bautista, A., Petisco Rodríguez, E. y Lara Gonzalo, P. (2009). Estudio sobre situación de Necesidades Sociosanitarias de las personas con Enfermedades Raras en España. Estudio ENSERio. Madrid: Federación Española de Enfermedades Raras FEDER. Accesible en:

https://www.enfermedades-raras.org/images/stories/documentos/Estudio ENSERio.pdf

Jensen, K. (2016). “Prosociality”. Current Biology 26(16): R748-R752. https:// doi.org/10.1016/j.cub.2016.07.025

Jiménez, F. (2011). Racionalidad pacifica. Una introducción a los Estudios para la paz. Madrid, Dykinson.

Jutel, A. (2006). "The emergence of overweight as a disease entity: Measuring up normality". Social Science \& Medicine 63(9): 2268-2276. https://doi. org/10.1016/j.socscimed.2006.05.028

Laudan, L. (1977). Progress and its Problems. Towards a Theory of Scientific Growth, Berkeley, Univ. of California Press.

Lévinas, E. (1987). De otro modo de ser, o más allá de la esencia, Sígueme, Salamanca.

López, M. C. y Chicano, G. (2017). "La integración laboral y social de colectivos especialmente vulnerables: personas con enfermedades raras, personas con enfermedades mentales y mujeres con discapacidad". En: OIT El futuro del trabajo que queremos. Conferencia Nacional Tripartita, 28 de marzo de 2017. Vol. II. Madrid: Ministerio de Trabajo y Seguridad Social: pp. 159-170

McNaughton, D., Rackensperger, T., Dorn, D. y Wilson, N. (2014). "Home is at work and work is at home: telework and individuals who use augmentative and alternative communication". Work, 48(1), 117-126

Mercader Uguina, J.R. (2016). "Ley de Prevención de Riesgos Laborales, veinte años después". Seguritecnia, 427:62-63

Morin, E. (1997). El pensamiento complejo. Madrid, Paidós.

Paramá, A. y Fernández, A.M. (2014). "La ética reticular y su aplicación en la enfermería”. Estudios filosóficos 63(184): 495-502.

Peirce, C. S. (1931-1958). Collected Papers, vols. 1-6, ed. C. Hartshorne and P. Weiss, vols. 7-8, ed. A.W. Burks. Cambridge, M.A., Harvard University Press.

Puyol, A. (2017). El derecho a la fraternidad. Madrid, Catarata.

Ramalle-Gómara, E., Ruiz, E., Quiñones, C., Andrés, S., Iruzubieta, J. y Gil-deGómez, J. (2015). "General knowledge and opinion of future health care and non-health care professionals on rare diseases". Journal of Evaluation on Clinical Practice 21(2), 198-201 
Rosenberg, C.E. (2002). "The tyranny of diagnosis". The Milbank Quarterly 80, 237-260.

Royzman, E.B. y Rozin, P. (2006). Limits of symhedonia: the differential role of prior emotional attachment in sympathy and sympathetic joy. Emotion (Washington, D.C.), 6(1), 82-93. https://doi.org/10.1037/1528-3542.6.1.82

Queraltó, R. (1999). "Cómo introducir vectores éticos eficaces en el sistema tecnológico", Arbor, 638, 221-240.

Queraltó, R. (2003). Ética, tecnología y valores en la sociedad global. El caballo de Troya al revés, Tecnos, Madrid.

Queraltó, R. (2008a), "Mutación de la ética en la sociedad tecnológica contemporánea. Ética y felicidad humana”, Ludus Vitalis XVI (30): 165196.

Queraltó, R. (2008b). La estrategia de Ulises o ética en una sociedad tecnológica, CICTES-DOSS, Madrid-Sevilla.

Queraltó, R. (2010). "Ética, racionalidad... y también tecnología. Tratando de atar algunos cabos", Ludus Vitalis, vol. XVIII, (33), 245-263.

Rose, N. (2001). "Normality and Pathology in a Biological Age". Outlines 1: 19-33.

Soldevilla, C. (2013). Ser barroco: una hermenéutica de la cultura. Madrid: Biblioteca Nueva.

Valero Matas, J.A. y Coca, J.R. (2012). "Identidad y cultura tecnocientíficas como factores potenciales de discriminación social", Aposta. Revista de ciencias sociales, $\mathrm{N}^{\circ}$ 55. Accesible en: http://www.apostadigital.com/ revistav3/hemeroteca/jcoca1.pdf Acceso: 23/06/14.

Vicente, M.T., Torres, J.I., Torres, A., Ramírez, M.V. y Capdevila, L. (2018). "El teletrabajo en salud laboral: Aspectos médico-legales y laborales". Revista CES Derecho 9 (2), 287-297.

Von der Lippe, C., Diesen, P.S. y Feragen, K.B. (2017). "Living with a rare disorder: a systematic review of the qualitative literature". Molecular Genetics \& Genomic Medicine 5(6), 758-773. https://doi.org/10.1002/ $\underline{\operatorname{mgg} 3.315}$

Zubizarreta, A.C., y Ruiz, R.G. (2014). "La escolarización de niños con enfermedades raras: Visión de las familias y del profesorado". REICE: Revista Electrónica Iberoamericana Sobre Calidad, Eficacia y Cambio En Educación 12(1), 119-135. 
